\title{
RANKL Inhibitor: A Future Chemo Preventive Agent for High Risk Breast Cancer
}

\author{
Aalekhya Sharma Biswas ${ }^{1}$, Abhijit Chakraborty ${ }^{2}$ \\ ${ }^{1}$ Dept. of Biotechnology, Maulana Abul Kalam Azad University of Technology, Kolkata, India \\ ${ }^{2}$ Cellular \& Molecular Biology Division, The Hormel Institute - University of Minnesota, USA \\ dr.chakrabortyabhijit@gmail.com
}

\begin{abstract}
Hereditary breast cancer constitutes 5-10\% of all breast cancer cases. Inherited mutations in the BRCA1 and BRCA2 tumour-suppressor genes, account for the majority of hereditary breast cancer cases. Individuals who have mutations in those gene usually undergone prophylactic mastectomy to minimize their risk of breast cancer. The identification of an effective prevention therapy will be a great invention in this field. Recent studies suggested that tumor necrosis factor super family member 11 (TNFSF11) which is also known as $R A N K L$, play an important role in progesterone signalling. The RANKL and its receptor TNFRSF11A which is also known as RANK, contribute to mammary tumorigenesis. RANKL/RANK function is essential for epithelial cell proliferation and cellular survival as well as lobulo-alveolar development. An over expression of RANK has been shown in mammary tumour cells which express a non functional BRCAl mutation and also enhanced acinar invasiveness and increased tumorigenesis. Inhibition of RANKL can be a targetable pathway to prevent mammary cell tumorigenesis and BRCA-mutated tumour. RANKL inhibitor will be a promising treatment strategy in the prevention of breast cancer in near future.
\end{abstract}

Keywords: RANKL, RANK, Inhibitor, BRCA mutation, Chemotherapeutic agents.

\section{INTRODUCTION}

Despite of making good progress in the medical field for diagnosis and treatment, cancer is still a big threat to our society. Breast cancer is the most common cancer among worldwide population. The most common malignancy among females, all over the world, is Breast Cancer (BC). BRCA gene mutations immensely increase the risk of developing breast cancer (BC) among women. World Health Organization (WHO) reported that the incidence of breast cancer is increasing by $2 \%$ per year. GLOBOCAN (WHO) reported that, in 2012, approximately 70218 women died in India due to breast cancer [1]. Near about 5-10\% of all BC cases and 10-15\% of all ovarian cancer (OC) cases are due to germline mutations in one of the two breast cancer susceptibility genes, BRCA1 and BRCA2 [2,3].

Germline mutations in $B R C A 1$ or $B R C A 2$ pathogenic variants have up to an $87 \%$ risk of developing cancer, while males have up to a $20 \%$ risk [4]. A high proportion as $84 \%$ has been reported in certain families [5]. BRCA proteins are involved in repair of DNA double strand breakage [6]. Loss of function in BRCA1 and BRCA2 genes is responsible for developing cancer. The mutations in both proteins also increase the risk of ovarian and prostate cancers [6].BRCA mutation gene induce the lack of expression of estrogen, progesterone, and HER2 that define the biologically aggressive Triple Negative Breast Cancers (TNBC) subtype which shows a poor prognosis [7]. BRCA1-related tumors show higher histo logic grade, high number of metastatic lymph node involvement and are more likely to be estrogen receptor-negative and progesterone receptor- negative [8]. The BRCA1 and BRCA2 defective tumors has a specific pattern of genetic alterations required for tumor genesis. Tumor suppressor BRCA1 inhibits MYC's transcriptional and transforming activity. Loss of BRCA1 with MYC over expression leads to the development of breast cancer [9]. P53 mutation also play a role in the proliferation defect of BRCA1- or BRCA2-deficient tumor [10]. So, the BRCA1 mutations in ER and PR negative tumours have poor response to tamoxifen therapy $[11,12]$.

Prophylactic bilateral mastectomy decreasing the risk of developing BC by approximately $95 \%$ of the patients with a BRCA1 mutation $[13,14]$. There is recent evidence that oophorectomy is an effective primary prevention option but only for BRCA2- but not BRCA1-associated breast cancer [15]. 
Studies support that role of progesterone signalling on the pathogenesis of breast cancer [16].Considering the uncontrolled level of the BRCA1 mutation inducing breast cancer, it is important to identifying a novel target for chemoprevention. Exploring the mechanism BRCA associated breast cancer development should translate into targeted chemoprevention.

ER, PgR, and HER2/neu have proven to be very successful biomarkers that accurately predict benefit to treatment with tamoxifen and trastuzumab, respectively. However, it has proven more difficult to identify further biomarkers that could help predict response to specific chemotherapy drugs to tailor current chemotherapy regimens in an effort to gain maximum benefit with minimal exposure to unnecessary drugs. Despite this, we feel that there is some evidence to suggest that RANKL inhibitor could be useful as a chemotherapy agent against BRCA specific tumour. Clinical evidence to date has suggested an increased benefit from DNA damage-based chemotherapy for patients with BRCA1 germline mutations. There is currently insufficient evidence to suggest that RANLK inhibitor can be a good option for treatment particularly in BRCA positive patient's patients; however, we feel that the use of RANKL- inhibitor as a treatment option should be examined more fully in prospective clinical trials, not only in breast cancer but also in other cancers where BRCA1 seems to play a role in the development of the tumors such as ovarian, prostate, and non-small cell lung cancer.

\section{RANKL/RANK}

Receptor activator of nuclear factor (NF)-kB ligand (RANKL) is a protein that in humans is encoded by the TNFSF11 gene. RANKL is known as a type II membrane protein and is a member of the tumor necrosis factor (TNF) super family [17]. TNFRSF11 present in Chromosome no. 13 at a position of 13q14. The protein RANK consists of 317 amino acids and the molecular weight is 35478 Da. RANKL help to generate osteoclasts from their myeloid precursors [18]. RANKL receptor, Osteoprotegerin (OPG) inhibits osteoclasts and acts as a physiological regulator of bone resorption by maintaining the RANKL activity [19]. The molecular mechanism of RANKL, RANK and OPG open to treat osteoclast genesis and help in bone remodelling and repair, immune cell function, lymph node development, thermal regulation, and mammary gland development.

Receptor Activator of Nuclear Factor $\kappa \mathrm{B}$ (RANK), also known as TRANCE Receptor or TNFRSF11A. It is a member of the tumor necrosis factor receptor (TNFR). RANK is the receptor for RANK-Ligand (RANKL) and part of the RANK/RANKL/OPG signalling pathway. This pathway regulates osteoclast differentiation and activation. RANKL and its receptor RANK were initially characterized as essential factors for osteoclast genesis. The gene TNFRSF11A present in Chromosome no. 18 at18q21position. The protein RANK consists of 616 amino acids and the molecular weight is $66034 \mathrm{Da}$ [20]. Interestingly, it binds to the clefts between the subunits of the TNFSF11 ligand trimer to form a heterohexamer. The bindings partners are TRAF1, TRAF2, TRAF3, TRAF5 and TRAF6. RANK is an essential mediator for osteoclast and lymph node development. Mutations at this locus have been associated with familial expansile osteolysis, autosomal recessive osteopetrosis, and Paget disease of bone.

\section{RANKL/RAnK/ Opg Pathway}

The signalling pathway of RANKL/RANK is well describe by Raju et al. 2011 [21]. RANKL is expressed by bone stromal cells of the osteoblast lineage. It mediates bone resorption [22]. Cells of the osteoblast lineage or activated $\mathrm{T}$ cells express at least three different subtypes of RANKL [23]. Among them two subtypes remain on the cell surface showing the capacity to bind their TNFhomology domains. RANKL bind with RANK and promote the multinucleated osteoclast precursor cells [24] then its differentiated into mature osteoclastic cells. This cells became resistance to cell death by inhibiting apoptosis [25]. The osteoclasts then activated resorb bone [24]. The OPG then binds to RANKL and prevent RANKL from activating its receptor RANK. Hence, it inhibits the catabolic effects of RANKL. The osteoclasts formation and activation was eventually stopped. So, the bone volume, density and strength are increased. In this way the RANKL/RANK/ OPG play the role in maintenance of homeostatic bone remodeling. OPG directly binds to RANKL hence the relative balance of the two actors is important for the fragile system to properly function. This phenomenon concerning the interactions of OPG-RANK-RANKL led to the hypothesis that any dysregulation occurring in a disease state including postmenopausal osteoporosis or cancer-induced bone destruction may involve the described pathophysiology. 


\section{RANKL AND BREAST CANCER}

From current research, it was established that the RANKL has a significant role to development of BRCA-associated breast cancer. RANKL and RANK both have roles in mammary gland development, mammary epithelial and stem cell proliferation, differentiation and survival. Progesterone receptor negative mice shows a similar osteoclast-independent effects on mammary morphology when compared to RANK or RANKL knockout mice [26]. RANK-/RANKL knock down mice shows dis functioning of lobulo-alveolar mammary epithelial structures which cause by the lactation defects. RANKL shows low expression levels in luminal and basal mammary epithelial cells. [27]. Normally at a mid-gestation pregnancy the lactation hormone level became high which massively increasing the breast milk producing cells. RANKL/RANK plays an essential rolein epithelial cell proliferation and cellular survival as well as lobulo-alveolar development [28]. These induces by a up regulation of RANKL expression.

The mammary gland is organized into two main cell typesnamely the luminal and myoepithelial lineage. Luminal cells can be subdivided into ductal and alveolar cells and are mainly responsible for the mammary secretion of fluids and nutrients. Myoepithelial lineage cells are located adjacent to the basement membrane can exert contractile functions, thereby guiding the milk through the epithelial tree. The mammary progenitor cells undergo proliferation and differentiation during each oestrus cycle. This stem cell numbers change during the course of each oestrus cycle, during pregnancy as well as during ageing, which is responsible for mammary gland to adapt to altered physiological states. Recent studies showed that RANKL inhibition significantly suppressed mammary tumorigenesis in BRCA1 mutated tumor[20,30]. BRCA1 regulate genes that are critical for normal differentiation of luminal mammary epithelial cells. Tao et al. [31] shows that deletion of BRCA1 results in a reduced repopulating frequency, which established BRCA1 as a positive regulator of mammary stem cells. It has been found that genome variations within the RANK locus were significantly associated with risk of developing BRCA1 mutations related breast cancer. Progenitor cell expansion and tumorigenesis controlled by RANKL/RANK [30]. From these results, a preventive strategy can be undertaken for treatment of breast cancer patients with BRCA1 mutation. BRCA1mutation disrupt the mammary growth in response to exogenous progesterone in virgin mice [32]. Poole et al [33] showed that tumor development in Brca1/p53 deficient mice could be prevented by the PR signalling pathway. These findings highlight the potential value for RANKL inhibition in BRCA1-associated cancers at the early stages of tumorigenesis.

\section{DisCuSSION}

BRCA1mutation carrier have a probability of about $80 \%$ for developing breast cancer, and BRCA2 mutations carriers have a chance to developing breast cancer of about $85 \%$. Currently invitro chemoprediction assays like Chemo Fx, MICK and EDR by different companies predict best chemoresponse of a cancer but do not take into account the genetic and signalling behaviour of cells. Currently in some third world countries, the status of BRCA1 and 2 are not well defined and some publications indicate the presence mutations other than the founder mutations in the specific population. We constantly seen on-responders to chemo-therapy and hypothesize that BRCA 1 and 2 mutation statuses will give us a clear idea about chemo-sensitivity of a patient. Information pertaining to chemo-response will go along way in managing breast cancer patients. Improvements in the treatment of breast cancer over the last few decades mean that overall mortality from the disease is falling [34]. However, despite these improvements, near about $40 \%$ of women diagnosed with metastatic cancer and eventually die. Besides surgery, this is to very important to determine which women will gain benefit from adjuvant therapies such as chemotherapy, radiotherapy, and hormonal therapies. Presently, treatment decisions are made based on histopathological tumor characteristics. Metastatic triple negative breast cancer is incurable; as because the main targets (ER/PR/Her2neu) to treat the disease is absent. There are many different treatment options available for women with metastatic disease, including chemotherapy, radiotherapy, and hormonaltherapy, and it is essential to give treatments that provide maximal benefit. Molecular markers currently used to predict treatment response in both early and metastatic breast cancer include estrogenreceptor (ER), progestogen receptor (PgR), and HER2/neu.

It has been reported by Metcalfe et al. in 2008 [35] that only 5.5\% of women suffering from breast cancer with a BRCA1 or BRCA2 mutation have used tamoxifen to reduce the risk of developing breast cancer. Till now most of the individuals at risk depends on screening for the early detection of 
breast cancer. The advantages and disadvantages of prophylactic hysterectomy have to be discussed in details with patients. Vicus et al. in 2009 [36] have reported in his study that in patients with BRCA1mutation and with a history of breast cancer, treatment with tamoxifen does not increase ovarian cancer risk. Fong et al. in 2009 [37] and Tutt et al. in 2010 [38] established in their clinical research study that PARP1 and PARP2 inhibitor is effective in treating advanced breast and ovarian cancer patients with BRCA1/2 germline mutation as well as in advanced breast cancer patients with BRCA1/2 mutations. A number of clinical trials are currently ongoing with the use of various PARP inhibitors, both as single agents and in combination with chemotherapy, for both hereditary and sporadic breast and ovarian cancer. None of these have proven reproducible enough to be used inroutine clinical practice; therefore, the search to find the chemotherapeutic agent that will the drug of choice to treatment of BRCA mediated breast cancer continues.

Recent studies [29] reported that when RANKL inhibitor like denosumab is used, the proliferation of organoids derived from BRCA1-mutation carriers was significantly reduced. After transplantation of mammary tumour in a mouse model, Nolan et al. [29] identified a significant RANKL-dependent reduction of tumour incidence. Denosumab, a monoclonal RANKL-blocking antibody was developed to treatment of osteoporosis. This can be used as a RANKL inhibitor for BRCA mutated breast cancer also. Apart from denosimab, new RANKL inhibitor need to identified which will be a future drug to treatment of BRCA related breast cancer.

\section{Conclusion}

As no standard drug exist to predict chemo-response in BRCA mutated patients, the RANKL can open up exciting determinants of therapeutic modalities. Upfront prediction of responders and nonresponders to defined drugs will enable clinicians to design therapy module according to the need of the patient, there by reducing unnecessary toxicity generated from non- working drugs, increase in time to recurrence, progression free survival and overall survival. The RANKL inhibitor may also point the way to new chemotherapeutic strategies targeting BRCA1-mediated pathways that participate in cancer cell death or an improved response to conventional treatments. Women with high risk group for developing breast cancer can be controlled using such drug. Determination of efficacy of denosumab in BRCA1-mutation carriers in clinical trials is need to be essential on urgent basic. The emerging role of aberrant RANK-signalling proved that RANKL inhibitor will be a promising drug to prevention of breast cancer in near future. It can also help to treat the Triple Negative Breast Cancer (TNBC) who containing BRCA mutation. RANKL inhibitors can reduce recurrence and metastasis in breast cancer patients based on its ability to induce tumor cell differentiation. Patients may quickly benefit from this therapeutic strategy to combat advanced breast cancer.

\section{REFERENCES}

[1] Ferlay J, Soerjomataram I, Ervik M et al. GLOBOCAN 2012 v1.0, Cancer Incidence and Mortality Worldwide: IARC Cancer Base No. 11 [Internet].

[2] Miki Y, Swensen J, Shattuck-Eidens D, Futreal PA, Harshman K, Tavtigian S, et al. A strong candidate for the breast and ovarian cancer susceptibility gene BRCA1. Science, 266:66-71 (1994).

[3] Wooster R, Bignell G, Lancaster J, Swift S, Seal S, Mangion J, et al. Identification of the breast cancer susceptibility gene BRCA2. Nature, 378:789-92 (1995).

[4] Petrucelli N, Daly MB, Pal T. BRCA1- and BRCA2-Associated Hereditary Breast and Ovarian Cancer. 1998 Sep 4 [Updated 2016 Dec 15]. In: Pagon RA, Adam MP, Ardinger HH, et al., editors. Gene Reviews ${ }^{\circledR}$ [Internet]. Seattle (WA): University of Washington, Seattle; 1993-2017. Available from: https://www.ncbi.nlm.nih.gov/books/NBK1247/.

[5] Antoniou A, Pharoah PD, Narod S, Risch HA, Eyfjord JE, Hopper JL, et al. Average risks of breast and ovarian cancer associated with BRCA1 or BRCA2 mutations detected incase series unselected for family history: a combined analysis of 22 studies. Am J Hum Genet. 72(5):111730 (2003).

[6] Biswas AS and Chakraborty A. BRCA1 and BRCA2 Mutation in Pancreatic Cancer: Significance in Therapeutic Approach. Austin J Gastroenterol. 4(1): 1076 (2017)

[7] Lips EH, Mulder L, Oonk A, van der Kolk LE, Hogervorst FBL, Imholz ALT, et al. Triplenegative breast cancer: BRCAness and concordance of clinical features with BRCA1-mutation carriers. Br J Cancer, 108:2172-7 (2013). 
[8] Chakraborty A, Bose CK, Basak J, Sen AN, Mishra R, Mukhopadhyay A. Determinants of lymph node status in women with breast cancer: A hospital based study from eastern India. The Indian Journal of Medical Research.143(Suppl 1):S45-S51 (2016).

[9] Wang Q, Zhang H, Kajino K, et al. BRCA1 binds c-Myc and inhibits its transcriptional and transforming activity in cells. Oncogene, 17:1939-1948 (1998).

[10] Evers B, Jonkers J. Mouse models of BRCA1 and BRCA2 deficiency: past lessons, current understanding and future prospects. Oncogene, 25(43):5885-5897 (2006).

[11] L. Moore-Smith and B. Pasche, "TGFBR1 signaling and breast cancer," Journal of Mammary Gland Biology and Neoplasia, vol. 16, no. 2, pp. 89-95, 2011.

[12] D. F. Easton, "How many more breast cancer predisposition genes are there?" Breast Cancer Research, vol. 1, no. 1, pp. 14-17, 1999.

[13] Hartmann LC, Schaid DJ, Woods JE, Crotty TP, Myers JL, Arnold PG, Petty PM, Sellers TA, Johnson JL, McDonnell SK, Frost MH, Jenkins RB. Efficacy of bilateral prophylactic mastectomy in women with a family history of breast cancer. N Engl J Med, 340(2):77-84 (1999).

[14] Hartmann LC, Sellers TA, Schaid DJ, Frank TS, Soderberg CL, Sitta DL, Frost MH, Grant CS, Donohue JH, Woods JE, McDonnell SK, Vockley CW, Deffenbaugh A, Couch FJ, Jenkins RB. Efficacy of bilateral prophylactic mastectomy in BRCA1 and BRCA2 gene mutation carriers. J Natl Cancer Inst 93(21):1633-1637 (2001).

[15] Kotsopoulos J, Huzarski T, Gronwald J, Singer CF, Moller P, Lynch HT, Armel S1, Karlan B, Foulkes WD, Neuhausen SL, Senter L, Tung N, Weitzel JN, Eisen A, Metcalfe K, Eng C, Pal T, Evans G, Sun P, Lubinski J, Narod SA2; Hereditary Breast Cancer Clinical Study Group. Bilateral Oophorectomy and Breast Cancer Risk in BRCA1 and BRCA2 Mutation Carriers. J Natl Cancer Inst. 109(1). pii: djw177 (2016).

[16] Chlebowski RT, Rohan TE, Manson JE, Aragaki AK, Kaunitz A, Stefanick ML, Simon MS, Johnson KC, Wactawski-Wende J, O'Sullivan MJ, Adams-Campbell LL, Nassir R, Lessin LS, Prentice RL. Breast cancer after use of estrogen plus progestin and estrogen alone: analyses of data from 2 women's health initiative randomized clinical trials. JAMA Oncol 1(3): 296-305 (2015).

[17] Hanada R, Hanada T, Sigl V, Schramek D, Penninger JM. RANKL/RANK-beyond bones. J. Mol. Med. 89 (7): 647-656 (2011).

[18] Lacey DL, Timms E, Tan HL, Kelley MJ, Dunstan CR, Burgess T et al. Osteoprotegerin ligand is a cytokine that regulates osteoclast differentiation and activation. Cell.93:165-176 (1998).

[19] Simonet WS, Lacey DL, Dunstan CR, Kelley M, Chang MS, Luthy R et al. Osteoprotegerin: a novel secreted protein involved in the regulation of bone density. Cell. 89:309-319 (1997).

[20] Anderson DM, Maraskovsky E, Billingsley WL, Dougall WC, Tometsko ME, Roux ER, et al. A homologue of the TNF receptor and its ligand enhanceT-cell growth and dendritic-cell function. Nature 390,175-179 (1997).

[21] Raju R, Balakrishnan L, Nanjappa V, Bhattacharjee M, Getnet D, Muthusamy B et al. A comprehensive manually curated reaction map of RANKL/RANK signaling pathway. Database (Oxford). 2011, bar021

[22] Wong B, Josien R, Lee SY, B. Sauter B, Li HL, Steinman RM, Choi Y, TRANCE (tumor necrosis factor [TNF]-related activationinduced cytokine), a new TNF family member predominantly expressed in $\mathrm{T}$ cells, is a dendritic cell-specific survival factor, J. Exp. Med. 186.2075-2080 (1997).

[23] Hikita A, Yana I, Wakeyama H, Nakamura M, Kadono Y, Oshima Y, et al. Negative regulation of osteoclastogenesis by ectodomain shedding of receptor activator of NF-B ligand, J Biol Chem. 281:36846-36855 (2006).

[24] Lacey DL, TimmsE, Tan HL, Kelley MJ, Dunstan CR, T. Burgess T, et al. Osteoprotegerin ligand is a cytokine that regulates osteoclast differentiation and activation, Cell. 93:165-176 (1998).

[25] Lacey DL, Tan HL, Lu J, Kaufman S, Van G,Qiu W, et al, Osteoprotegerin ligand modulates murine osteoclast survival in vitro and in vivo, Am J Pathol. 157:435-448 (2000). 
[26] Karsenty G. The genetic transformation of bone biology Genes Dev. 13:3037-3051 (1999).

[27] Gonzalez-SuareE, Branstetter D, Armstrong A, Dinh H, Blumberg H, Dougall WC. RANK over expression in transgenic mice with mouse mammary tumor virus promoter controlled RANK increases proliferation and impairs alveolar differentiation in the mammary epithelia and disrupts lumen formation in cultured epithelial acini. Mol Cell Biol. 27 (4):1442-1454 (2007).

[28] Fata JE, Kong YY, Li J, Sasaki T, Irie-Sasaki J, Moorehead RA, et al. The osteoclast differentiation factor osteoprotegerin-ligand is essential for mammary gland development Cell, 103: 41-50 (2000).

[29] Nolan E, Vaillant F, Branstetter D, Pal B, Giner G, Whitehead L, Lok SW, Mann GB; Kathleen Cuningham Foundation Consortium for Research into Familial Breast Cancer (kConFab), Rohrbach K, Huang LY, Soriano R, Smyth GK, Dougall WC, Visvader JE, Lindeman GJ. RANK ligand as a potential target for breast cancer prevention in BRCA1-mutation carriers. Nat Med.22 (8):933-939 (2016).

[30] Sigl V, Owusu-Boaitey K, Joshi PA, Kavirayani A, Wirnsberger G, Novatchkova M, Kozieradzki I, Schramek D, Edokobi N, Hersl J, Sampson A, Odai-Afotey A, Lazaro C, Gonzalez-Suarez E, Pujana MA, Cimba F, Heyn H, Vidal E, Cruickshank J, Berman H, Sarao R, Ticevic M, Uribesalgo I, Tortola L, Rao S, Tan Y, Pfeiler G, Lee EY, Bago-Horvath Z, Kenner L, Popper H, Singer C, Khokha R, Jones LP, Penninger JM. RANKL/RANK control Brca1 mutation-driven mammary tumors. Cell Res. 26(7):761-74 (2016).

[31] Tao L, Roberts AL, Dunphy KA, Bigelow C, Yan H, Jerry DJ. Repression of mammary stem/ progenitor cells by p53 is mediated by notch and separable from apoptotic activity. Stem Cells. 29,119- 127 ( 2011).

[32] Ma Y, Katiyar P, Jones LP, et al. The breast cancer susceptibility gene BRCA1 regulates progesterone receptor signaling in mammary epithelial cells. MolEndocrinol; 20:14-34 (2006).

[33] Poole AJ, Li Y, Kim Y, Lin SC, Lee WH, Lee EY. Prevention of Brca1-mediated mammary tumorigenesis in mice by a progesterone antagonist. Science. 314:1467-1470 (2006).

[34] Brown P. UK death rates from breast cancer fall by a third. BMJ. 321(7265):849 (2000).

[35] Metcalfe KA, Birenbaum-Carmeli D, Lubinski J, et al. International variation in rates of uptake of preventive options in BRCA1 and BRCA2 mutation carriers. Int J Cancer. 122:2017-2022 (2008).

[36] Vicus D, Rosen B, Lubinski J, et al. Tamoxifen and the Risk of Ovarian Cancer in BRCA1 Mutation Carriers. GynecolOncol. 115: 135-137 (2009).

[37] Fong PC, Boss DS, Yap TA, et al. Inhibition of poly(ADP-ribose) polymerase in tumors from BRCA mutation carriers. N Engl J Med. 361:123-134 (2009).

[38] Tutt A, Robson M, Garber JE, et al. Oral poly(ADP-ribose) polymerase inhibitor olaparib in patients with BRCA1 or BRCA2 mutations and advanced breast cancer: a proof-of-concept trial. Lancet. 376:235-244 (2010). 